Article

\title{
On Sliced Spaces: Global Hyperbolicity Revisited
}

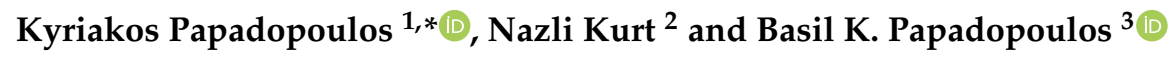 \\ 1 Department of Mathematics, Kuwait University, PO Box 5969, Safat 13060, Kuwait \\ 2 Faculty of Science, Open University, PO Box 197, Milton Keynes MK7 6BJ, UK; nazkrt.96@gmail.com \\ 3 Department of Civil Engineering, Democritus University of Thrace, 67100 Xanthi, Greece; \\ papadob@civil.duth.gr \\ * Correspondence: kyriakos.papadopoulos1981@gmail.com
}

Received: 24 January 2019; Accepted: 26 February 2019; Published: 1 March 2019

check for updates

\begin{abstract}
We give a topological condition for a generic sliced space to be globally hyperbolic without any hypothesis on lapse function, shift function, and spatial metric.
\end{abstract}

Keywords: sliced space; product topology; Alexandrov topology; global hyperbolicity

\section{Preliminaries}

The definition of a sliced space, which one can read in Reference [1], is a continuation of a study in References [2] and [3] on systems of Einstein equations.

Let $V=M \times I$, where $M$ is an $n$-dimensional smooth manifold, and $I$ is an interval of the real line, $\mathbb{R}$. We equip $V$ with a $n+1$-dimensional Lorentz metric $g$, which splits in the following way:

$$
g=-N^{2}\left(\theta^{0}\right)^{2}+g_{i j} \theta^{i} \theta^{j},
$$

where $\theta^{0}=d t, \theta^{i}=d x^{i}+\beta^{i} d t, N=N\left(t, x^{i}\right)$ is the lapse function, $\beta^{i}\left(t, x^{j}\right)$ is the shift function and $M_{t}=M \times\{t\}$, spatial slices of $V$, are spacelike submanifolds equipped with the time-dependent spatial metric $g_{t}=g_{i j} d x^{i} d x^{j}$. Such product space $V$ is called a sliced space.

Throughout the paper, we consider $I=\mathbb{R}$.

The author in Reference [1] considered sliced spaces with uniformly bounded lapse, shift, and spatial metric; by this hypothesis, it is ensured that parameter $t$ measures up to a positive factor bounded (below and above) the time along the normals to spacelike slices $M_{t}$, the $g_{t}$ norm of the shift vector $\beta$ is uniformly bounded by a number, and the time-dependent metric $g_{i j} d x^{i} d x^{j}$ is uniformly bounded (below and above) for all $t \in I(=\mathbb{R})$, respectively.

Given the above hypothesis, in the same article, the following theorem was proved.

Theorem 1 (Cotsakis). Let $(V, g)$ be a sliced space with uniformly bounded lapse $N$, shift $\beta$ and spatial metric $g_{t}$. Then, the following are equivalent:

1. $\left(M_{0}, \gamma\right)$ a complete Riemannian manifold.

2. Spacetime $(V, g)$ is globally hyperbolic.

In this article, we review global hyperbolicity of sliced spaces in terms of the product topology defined on space $M \times \mathbb{R}$ for some finite dimensional smooth manifold $M$.

\section{Strong Causality of Sliced Spaces}

Let $(V=M \times \mathbb{R}, g)$ be a sliced space. Consider product topology $T_{P}$ on $V$. Since $M$ is finite-dimensional, a base for $T_{P}$ consists of all sets of form $A \times B$, where $A \in T_{M}$ and $B \in T_{\mathbb{R}}$. 
Here, $T_{M}$ denotes the natural topology of manifold $M$ where, for an appropriate Riemann metric $h$, it has a base consisting of open balls $B_{\epsilon}^{h}(x)$, and $T_{\mathbb{R}}$ is the usual topology on the real line, with a base consisting of open intervals $(a, b)$. For trivial topological reasons, we can restrict our discussion on $T_{P}$ to basic-open sets $B_{\epsilon}^{h}(x) \times(a, b)$, which can intuitively be called "open cylinders" in $V$.

We remind that the Alexandrov topology $T_{A}$ (see Reference [4]) has a base consisting of open sets of the form $\langle x, y\rangle=I^{+}(x) \cap I^{-}(y)$, where $I^{+}(x)=\{z \in V: x \ll z\}$ and $I^{-}(y)=\{z \in V: z \ll y\}$, where $\ll$ is the chronological order defined as $x \ll y$ iff there exists a future-oriented timelike curve joining $x$ with $y$. By $J^{+}(x)$, one denotes the topological closure of $I^{+}(x)$, and by $J^{-}(y)$ that one of $I^{-}(y)$.

We use the definition of global hyperbolicity from Reference [4], where one can read about global causality conditions in more detail, as well as characterizations for strong causality. In particular, a spacetime is strongly causal iff it possesses no closed timelike curves, and global hyperbolicity is an important causal condition in a spacetime related to major problems such as spacetime singularities and cosmic cencorship.

Definition 1. A spacetime is globally hyperbolic iff it is strongly causal and the "causal diamonds" $J^{+}(x) \cap J^{-}(y)$ are compact.

We prove the following theorem:

Theorem 2. Let $(V, g)$ be a Hausdorff sliced space. Then, the following are equivalent.

1. $\quad V$ is strongly causal.

2. $T_{A} \equiv T_{P}$.

3. $T_{A}$ is Hausdorff.

Proof. Here, 2. implies 3. is obvious and that 3. implies 1. can be found in Reference [4].

For 1. implies 2., we consider two events $X, Y \in V$, such that $X \neq Y$; we note that each $X \in V$ has two coordinates, say $\left(x_{1}, x_{2}\right)$, where $x_{1} \in M$ and $x_{2} \in \mathbb{R}$. Obviously, $X \in M_{x}=M \times\{x\}$ and $Y \in M_{y}=M \times\{y\}$. Then, $<X, Y>=I^{+}(X) \cap I^{-}(Y) \in T_{A}$. Let also $A \in M_{a}=M \times\{a\}$, where $a<x$ ( $<$ is the natural order on $\mathbb{R}$ ) and $B \in M_{b}=M \times\{b\}$, where $y<b$. Consider some $\epsilon>0$, such that $B_{\epsilon}^{h}(A) \in M$. Obviously, $B_{\epsilon}^{h}(A) \times(a, b) \in T_{P}$ and, for $\epsilon>0$ sufficiently large enough, $<X, Y>\subset B_{\epsilon}^{h}(A) \times(a, b)$. Thus, $<X, Y>\in T_{P}$.

For 2. implies 1., we consider $\epsilon>0$, such that $B_{\epsilon}^{h}(A) \in T_{M}$, so that $B_{\epsilon}^{h}(A) \times(a, b)=B \in T_{P}$. We let strong causality hold at an event $P$ and consider $P \in B \in T_{P}$. We show that there exists $\langle X, Y\rangle \in T_{A}$, such that $P \in\langle X, Y\rangle \subset B$. Now, consider a simple region $R$ in $\langle X, Y\rangle$ which contains $P$ and $P \in Q$, where $Q$ is a causally convex-open subset of $R$. Thus, we have $U, V \in Q$, such that $P \in\langle U, V>\subset Q$. Finally, $P \in\langle U, V>\subset Q \subset B$, and this completes the proof.

\section{Global Hyperbolicity of Sliced Spaces, Revisited}

For the following theorem, we use Nash's result that refers to finite-dimensional manifolds (see Reference [5]).

Theorem 3. Let $(V, g)$ be a Hausdorff sliced space, where $V=M \times R, M$ is an n-dimensional manifold and $g$ the $n+1$ Lorentz metric in $V$. Then,$(V, g)$ is globally hyperbolic iff $T_{P}=T_{A}$, in $V$.

Proof. Given the proof of Theorem 2, strong causality in $V$ holds iff $T_{P}=T_{A}$ and, given Nash's theorem, the closure of $B_{\epsilon}^{h}(x) \times(a, b)$ is compact.

We note that neither in Theorem 2 nor in Theorem 3 did we make any hypothesis on the lapse function, shift function, or spatial metric. 
Author Contributions: Conceptualization, K.P. and N.K.; methodology, K.P., N.K. and B.K.P.; investigation, K.P. and N.K.; resources, K.P.; writing—original draft preparation, K.P.; writing—review and editing, K.P.; supervision, K.P. and B.K.P.; project administration, K.P. and N.K.

Funding: This research received no external funding.

Conflicts of Interest: The authors declare no conflict of interest.

\section{References}

1. Cotsakis, S. Global hyperbolicity of sliced spaces. Gen. Rel. Grav. 2004, 36, 1183-1188. [CrossRef]

2. Choquet-Bruhat, Y.; Ruggeri, T. Hyperbolicity of the $3+1$ system of Einstein equations. Com. Math. Phys. 1983, 89, 269-275. [CrossRef]

3. Choquet-Bruhat, Y.; Cotsakis, S. Global hyperbolicity and completeness. J. Geom. Phys. 2002, 43, 345-350. [CrossRef]

4. Penrose, R. Techniques of Differential Topology in Relativity; Society for INdustrial and Applied Mathematics: PhiladeIphia, PA, USA, 1972, ISBN 978-0-898710-05-2.

5. Nash, J. $C^{1}$ isometric imbeddings. Ann. Math. Second Ser. 1954, 60, 383-396. [CrossRef]

(c) 2019 by the authors. Licensee MDPI, Basel, Switzerland. This article is an open access article distributed under the terms and conditions of the Creative Commons Attribution (CC BY) license (http:/ / creativecommons.org/licenses/by/4.0/). 